\title{
Trends in Haemophilus influenzae type b infections in adults in England and Wales: surveillance study
}

\author{
Jodie McVernon, Caroline L Trotter, Mary P E Slack, Mary E Ramsay
}

\begin{abstract}
Objective To describe invasive Haemophilus influenzae type $\mathrm{b}(\mathrm{Hib})$ infections in individuals aged 15 years or older in England and Wales between 1991 and 2003. Design Prospective, laboratory based surveillance of invasive Hib infections and cross sectional seroprevalence study.

Setting England and Wales.

Participants Cases were confirmed by isolation of $H$ influenzae from a normally sterile site, or from a non-sterile site in cases with a diagnosis of epiglottitis. Excess serum samples collected from English 30-39 year olds as part of a national serosurvey were identified for the years 1990, 1994, 1997, 2000, and 2002.

Main outcome measures The number of invasive $\mathrm{Hib}$ infections from 1991 to 2003. Population immunity to $H$ influenzae type b in English adults was also measured. Results After routine infant immunisation was introduced in October 1992, adult Hib infections decreased initially but then rose from a low in 1998 to reach prevaccine levels in 2003. An associated fall in median Hib antibody concentrations occurred, from $1.29 \mu \mathrm{g} / \mathrm{ml}(95 \%$ confidence interval 0.90 to 1.64$)$ in 1991 to $0.70 \mu \mathrm{g} / \mathrm{ml}(0.57$ to 0.89$)$ in $1994(\mathrm{P}=0.006)$, with no significant change observed thereafter.

Conclusions Although immunisation of infants resulted in an initial decline in Hib infections in adults, a resurgence in reported cases occurred in 2002-3. This rise was associated with an increase in cases in children and evidence of reduced immunity in older unimmunised cohorts. Childhood immunisation programmes may have unanticipated effects on the epidemiology of disease in older age groups, and surveillance strategies must be targeted at entire populations.
\end{abstract}

\section{Introduction}

Before routine vaccination was introduced, Haemophilus influenzae type b (Hib) was a notable cause of paediatric morbidity in England and Wales, with an annual incidence of invasive infection of about 30 per 100000 in children under 5 years of age. ${ }^{1}$ The inclusion of Hib conjugate vaccines in the routine immunisation schedule in October 1992 was associated with a rapid decline in reports of Hib disease. Ten years later, however, infections in children due to this pathogen increased markedly in England and Wales ${ }^{2}$ despite consistently high coverage in infants. ${ }^{1}$

Since 1998 the number of Hib cases in children has almost doubled each year, most of them in children who have been fully immunised according to the United Kingdom's primary vaccination schedule for infants aged 2, 3, and 4 months. ${ }^{2}$ Contributing factors include lower than anticipated direct protection from infant vaccination, ${ }^{3}$ wearing off of the initial impact of the "catch up" campaign, ${ }^{4}$ and the use of less immuno- genic, combination vaccines with an acellular pertussis component during 2000-1. ${ }^{5}$ With the decrease in Hib transmission attributed to use of the conjugate vaccine in the mid-1990s, cases in unimmunised individuals aged 15 years and older also declined, ${ }^{6}$ presumably as a result of indirect protection or herd immunity. We describe a reduction of this indirect effect of vaccination, as evidenced by a recent increase in invasive Hib infections in adults to prevaccine levels.

\section{Methods}

The Health Protection Agency identified cases through referral of $\mathrm{H}$ influenzae isolates to the Haemophilus Reference Unit and from reports of confirmed infections to the Communicable Disease Surveillance Centre from laboratories in England and Wales. We included cases if a strain of $H$ influenzae had been cultured from a normally sterile site, or from another site but with a clinical diagnosis of epiglottitis. For strains sent to the reference unit, the serotype was confirmed by polymerase chain reaction; for isolates that were not referred we used the typing results of the source laboratory. We used population denominators published by the Office for National Statistics to calculate age specific incidence for $H$ influenzae for England and Wales. We standardised these denominators to a 1991 population structure to account for any population changes over this period.

Between 1990 and 1995, five regions representing $35 \%$ of the English population (East Anglia, Northern, North West, South West, and Oxford) participated in an enhanced regional survey, involving referral of all strains to the Haemophilus Reference Unit. From late 1995, the reference unit followed up reports to the Communicable Disease Surveillance Centre from all English laboratories, or from public health doctors and treating clinicians, to obtain an isolate, where possible. To correct for changes in the proportion of isolates typed over time in the national dataset, we used the following formula:

$$
\begin{aligned}
& \text { Adjusted Hib reports }=\text { Known Hib reports }+ \\
& {\left[\left(\frac{\text { Known Hib }}{\text { All typed }}\right) \times \text { Untyped }\right]}
\end{aligned}
$$

The Health Protection Agency's seroepidemiology unit collects anonymous excess serum from hospital laboratories around the country each year. ${ }^{7}$ Sera taken from individuals with known immunodeficiency are excluded. We identified some 100 samples taken from 30-39 year old men and women for each of the years $1991,1994,1997,2000$, and 2002. None of these adults would have been eligible for routine vaccination with Hib conjugate vaccine. The immunoassay laboratory, Health Protection Agency Porton Down, used a standard HbO-HA ELISA to test the sera for antibodies to the Hib capsular polysaccharide polyribosyl-ribitolphosphate (PRP). ${ }^{8}$ Because of the highly skewed
Immunisation

Department, Health Protection Agency Communicable

Disease

Surveillance Centre, London NW9 5EQ

Jodie McVernon specialist registrar Mary E Ramsay consultant epidemiologist

Statistics, Modelling and Economics

Department, Health Protection Agency

Communicable

Disease

Surveillance Centre

Caroline L Trotter scientist (epidemiology)

Health Protection Agency Specialist and Reference

Microbiology

Division,

Haemophilus Reference Unit, John Radcliffe Hospital, Oxford OX3 9DU

Mary P E Slack consultant microbiologist

Correspondence to: M Ramsay mary.ramsay@ hpa.org.uk

BMJ 2004;329:655-8 
Table 1 Numbers (percentages) of laboratory reported invasive isolates in adults (15 years or older) by typing status and serotype, combined dataset from Haemophilus Reference Unit and Communicable Disease Surveillance Centre, 1991-2003

\begin{tabular}{lccrrr}
$\begin{array}{l}\text { Year of } \\
\text { report }\end{array}$ & $\begin{array}{c}\text { Haemophilus } \\
\text { influenzae type b }\end{array}$ & $\begin{array}{c}\text { Non-capsulate } \\
\text { Haemophilus } \text { strains }\end{array}$ & $\begin{array}{c}\text { Other capsular } \\
\text { serotypes }\end{array}$ & $\begin{array}{c}\text { Untyped } \\
\text { strains }\end{array}$ & Total \\
\hline 1991 & $68(30)$ & $32(14)$ & $4(2)$ & $122(54)$ & 226 \\
\hline 1992 & $71(33)$ & $39(18)$ & $10(5)$ & $92(43)$ & 212 \\
\hline 1993 & $63(23)$ & $71(26)$ & $12(4)$ & $131(47)$ & 277 \\
\hline 1994 & $35(16)$ & $71(33)$ & $6(3)$ & $101(47)$ & 213 \\
\hline 1995 & $35(14)$ & $121(47)$ & $18(7)$ & $85(33)$ & 259 \\
\hline 1996 & $16(7)$ & $105(46)$ & $28(12)$ & $78(34)$ & 227 \\
\hline 1997 & $29(11)$ & $121(44)$ & $22(8)$ & $101(37)$ & 273 \\
\hline 1998 & $13(5)$ & $149(55)$ & $17(6)$ & $94(34)$ & 273 \\
\hline 1999 & $32(11)$ & $142(49)$ & $14(5)$ & $102(35)$ & 290 \\
\hline 2000 & $32(10)$ & $157(48)$ & $21(6)$ & $119(36)$ & 329 \\
\hline 2001 & $45(13)$ & $171(50)$ & $24(7)$ & $101(30)$ & 341 \\
\hline 2002 & $99(25)$ & $174(44)$ & $28(7)$ & $98(25)$ & 399 \\
\hline 2003 & $118(28)$ & $182(43)$ & $38(9)$ & $86(20)$ & 424 \\
\hline Total & 656 & 1535 & 242 & 1310 & 3743 \\
\hline
\end{tabular}

distribution of antibody concentrations even after log transformation, we used medians as measures of central tendency for comparison over time. We used the binomial method to calculate $95 \%$ confidence intervals for the medians. We used a continuity corrected $\chi^{2}$ test in Stata, version 7, to assess the differences in median titres between consecutive time points.

\section{Results}

The reference laboratory received 3743 reports of invasive $H$ influenzae infections in adults over the period 1991-2003, of which 656 (18\%) were type b (Hib), 1535 (40\%) non-capsulate (ncHi), and 1310 (35\%) untyped (table 1). This number included 10 cases of Hib infection where the isolate was from a non-sterile site with a clinical diagnosis of epiglottitis. The proportion of adult $H$ influenzae isolates for which serotype was known improved from just under half in 1991 to $80 \%$ in 2003 (table 1). Much of this change can be attributed to increased referral of strains to the reference unit over the period, which rose from $41 \%$ to $88 \%$.

The most common clinical presentations of invasive Hib infection in adults were pneumonia $(n=142,22 \%)$, bacteraemia $(n=140,21 \%)$, epiglottitis

Table 2 Annual incidence of Hib disease per 100000 in adults aged 15 years and older (standardised to 1991 population) and in children younger than 5 years, combined dataset from Haemophilus Reference Unit and Communicable Disease Surveillance Centre, 1991-2003

\begin{tabular}{cccccc} 
& \multicolumn{2}{c}{ Adults $(\geq \mathbf{1 5}$ years) } & & \multicolumn{2}{c}{ Children $<\mathbf{5}$ years } \\
\cline { 2 - 3 } \cline { 5 - 6 } Year & $\begin{array}{c}\text { Incidence } \\
\text { per } \mathbf{1 0 0} \mathbf{0 0 0}\end{array}$ & $\mathbf{9 5 \%} \mathbf{~ C l}$ & & $\begin{array}{c}\text { Incidence } \\
\text { per } \mathbf{1 0 0} \mathbf{~ 0 0 0}\end{array}$ & $\mathbf{9 5 \% ~} \mathbf{~ I ~}$ \\
\hline 1991 & 0.17 & 0.13 to 0.21 & & 22.91 & 21.34 to 24.57 \\
\hline 1992 & 0.17 & 0.14 to 0.22 & & 21.91 & 20.38 to 23.53 \\
\hline 1993 & 0.15 & 0.12 to 0.19 & & 6.23 & 5.43 to 7.13 \\
\hline 1994 & 0.08 & 0.06 to 0.12 & & 1.08 & 0.76 to 1.49 \\
\hline 1995 & 0.08 & 0.06 to 0.12 & & 0.97 & 0.67 to 1.37 \\
\hline 1996 & 0.04 & 0.02 to 0.06 & & 0.90 & 0.61 to 1.29 \\
\hline 1997 & 0.07 & 0.05 to 0.10 & & 0.88 & 0.59 to 1.27 \\
\hline 1998 & 0.03 & 0.02 to 0.05 & & 0.65 & 0.40 to 0.99 \\
\hline 1999 & 0.08 & 0.05 to 0.11 & & 1.03 & 0.71 to 1.44 \\
\hline 2000 & 0.07 & 0.05 to 0.11 & & 1.93 & 1.47 to 2.47 \\
\hline 2001 & 0.10 & 0.08 to 0.14 & 3.01 & 2.43 to 3.69 \\
\hline 2002 & 0.23 & 0.19 to 0.29 & 4.63 & 3.89 to 5.46 \\
\hline 2003 & 0.27 & 0.23 to 0.34 & 3.90 & 3.23 to 4.67 \\
\hline
\end{tabular}

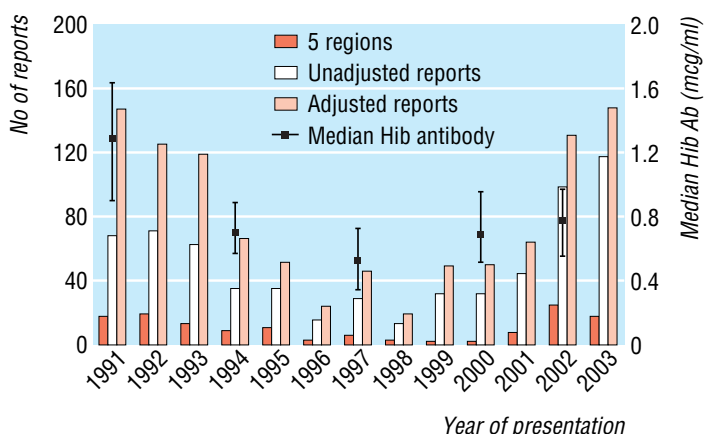

Fig 1 Number of Hib reports in adults (15 years or older) and median Hib antibody titres in people aged 30-39 years, by year

$(\mathrm{n}=106,16 \%)$, and meningitis $(\mathrm{n}=44,7 \%)$, together constituting two thirds of all reports. The outcome of Hib disease was known for $76 \%$ of adult cases reported between 1991 and 2003; the case fatality rate during each year ranged from 0 to $27 \%$ and was $11 \%$ in 2003 . Information on underlying medical conditions has been collected by the health protection Agency since 1996 , and such conditions were reported in 18/90 (20\%) of Hib cases in 1996-9 and 45/294 (15\%) of cases in 2000-3.

Adult infections due to serotype $b$ fell after the Hib vaccine was introduced in October 1992, reaching a low in 1998 and then rising to 2003 (table 1). This trend also became obvious in cases from the five regions conducting enhanced surveillance throughout the period and in the national dataset after adjusting for untyped isolates by using the method described above (fig 1). The incidence in all adults (15 years and older) decreased significantly between 1994 and 2000, followed by an increase in 2002 and 2003, which accompanied the changes in incidence in childhood (table 2).

Among adult cases with a known serotype, the age distribution differed between Hib and ncHi infections (fig 2). Whereas ncHi was predominantly a cause of serious infections in elderly people, the distribution in Hib cases was bimodal, peaking at 30 years and 65 years, ages at which household exposure to children is common. ${ }^{9}$

Antibody concentrations to $H$ influenzae type $\mathrm{b}$ in 30-39 year old English adults, collected at five time points over the period, showed a trend that was in keeping with the numbers of reports (fig 1). Hib

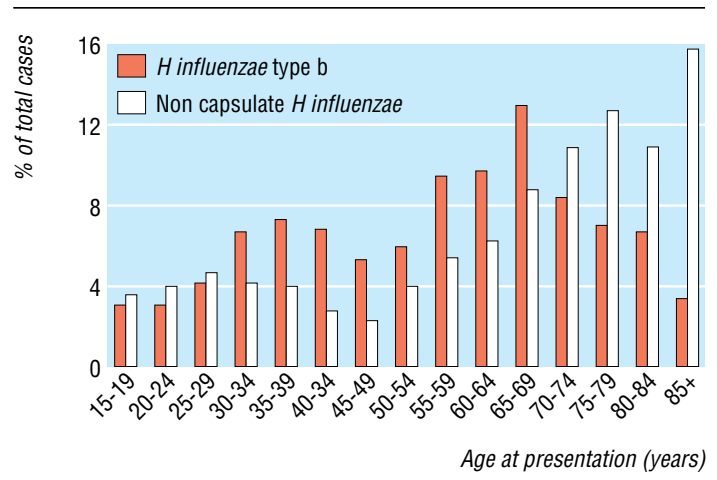

Fig 2 Age distribution of adults aged 15 or older with invasive disease caused by $H$ influenzae type $b$ and non-capsulate $H$ influenzae, 1991-2003 
antibody titres fell significantly, from $1.29 \mu \mathrm{g} / \mathrm{ml}(95 \%$ confidence interval 0.90 to 1.64 ) in 1991 to $0.70 \mu \mathrm{g} / \mathrm{ml}$ (0.57 to 0.89$)$ in $1994(\mathrm{P}=0.006)$, with a further non-significant decrease to $0.53 \mu \mathrm{g} / \mathrm{ml}(0.35$ to 0.73$)$ in 1997. A small, non-significant increase to $0.69 \mu \mathrm{g} / \mathrm{ml}$ (0.52 to 0.95$)$ occurred in $2000(\mathrm{P}=0.197)$, with another slight rise to $0.77 \mu \mathrm{g} / \mathrm{ml}(0.56$ to 0.97$)$ in $2002(\mathrm{P}=0.557)$.

\section{Discussion}

After Hib vaccine was introduced in October 1992 the number of cases of invasive Hib disease among adults in England and Wales fell markedly. This phenomenon was attributed to interruption of transmission of the organism from immunised children, or herd immunity. ${ }^{10}$ A resurgence in adult cases of invasive $\mathrm{Hib}$ disease occurred in 2002 and 2003, mirroring trends in paediatric infections over the same period and following a decline in the concentration of serum antibody to Hib in English adults aged 30-39 years. The fall in serum antibody concentrations may have resulted from reduced transmission of the organism, providing fewer opportunities for natural boosting of immunity.

The data presented here show how difficult it is to ensure consistency of case ascertainment over long periods of time and how important it is to account for this in the analysis. Improved reporting and typing led to an apparent increase in the incidence of invasive ncHi disease, ${ }^{611}$ but more constant numbers in recent years show that ascertainment has been stable since 1995. On the basis of unadjusted data, the number of adult Hib infections in recent years is higher than that observed in 1991. When the enhanced regional surveillance data are used, however, and after adjusting national figures for improved ascertainment, the current incidence in adults is similar to the incidence before the vaccine was introduced.

We took serum used to measure immunity to Hib in the population from a national serosurvey resource that collects residual sera from participating laboratories. $^{7}$ These specimens are stored anonymously, with limited linked information. Although it is possible that changes in selection of serum may have produced bias in the results obtained, comparison of a similar collection of sera in Australia showed no major discrepancy in antibody titres to common vaccine antigens with that from a random cluster survey. ${ }^{12}$

In contrast to ncHi infection, adults of the ages most likely to mix with children, both as parents and grandparents, seem to be most liable to Hib infections (fig 2). This observation is not surprising, given the high documented rates of parental carriage in families with a child known to be colonised with $H$ influenzae type $b$ or recovering from invasive Hib disease. ${ }^{13-15}$ It may therefore be predicted that an increase in paediatric Hib infections might be associated with a corresponding rise in adult cases. Of the 11 countries participating in the European Union Invasive Bacterial Infections Surveillance Network (EU-IBIS), only the Netherlands has noted a similar rise in adult Hib cases, from eight reports in 2001 to 15 in $2002,{ }^{16}$ the same number observed before the vaccine was introduced in 1993 (source: National Reference Laboratory for Bacterial Meningitis, Netherlands). This increase accompanies an increase in paediatric reports that has been observed despite a vaccine programme that does not

\section{What is already known on this topic}

Introduction of Hib conjugate vaccines in England and Wales led to a fall in invasive disease in all age groups, including those not eligible for vaccination, through indirect protection or "herd immunity"

\section{What this study adds}

A recent increase in invasive Hib infections in adults aged 15 years and older in England and Wales is approaching prevaccination levels and is associated with evidence of reduced antibody concentrations in older age groups

This increase may be due to reduced transmission of the organism and fewer opportunities for natural boosting of immunity by Hib colonisation

The unanticipated reduction in herd immunity highlights the need for surveillance of vaccine preventable diseases to be conducted across all age groups

use combination vaccines and where a booster is given in the second year of life. ${ }^{17}$ Although we believe that transmission is most often from child to parent, the reverse may also occur ${ }^{18}$ and may have contributed to some of the increase in invasive disease observed in children in recent years.

Our data show that the reduction in opportunities for natural boosting of immunity has resulted in a decline in specific Hib antibody titres among adults. Mathematical models of Hib transmission predicted that such a decline may lead to an increase in disease in the older unvaccinated population several years after the vaccine was introduced. ${ }^{19}$ The clinical presentation of or the case fatality due to Hib disease have not changed, and there is no indication that an increase in the prevalence of underlying conditions that predispose to invasive bacterial infection has contributed to this rise.

The high quality of surveillance in England and Wales allowed early detection of an increase in paediatric Hib cases, resulting in the withdrawal of poorly immunogenic vaccines and implementation of a national immunisation programme for children younger than 4 years. ${ }^{20}$ We anticipate that this campaign will rapidly induce herd immunity and prevent any further increase in infections in all age groups. Evaluation of the potential need for change to the routine immunisation schedule is ongoing and needs to include assessments of direct and indirect vaccine effects. Our findings may also be relevant to control of meningococcal and pneumococcal disease, where herd immunity effects in older unvaccinated age groups have also been observed after the conjugate vaccine was given to children. ${ }^{21}{ }^{22}$ It will be important to monitor whether such levels of indirect vaccine protection are sustained and this work emphasises the importance of maintaining surveillance of these infections across the entire age spectrum in the longer term.

We thank all of the microbiologists and clinicians who have reported cases of invasive Hib over the past 10 years. Particular thanks are due to Sue Gurney of the Haemophilus Reference Unit for administrative assistance and Suzanna Stringer for carrying out the laboratory procedures. We also thank Louise 
Hesketh, Andrew Vyse, and Elizabeth Miller of the HPA seroepidemiology unit for providing the samples for this study. The antibody testing was carried out at HPA Porton Down and we thank Moya Burrage, Lorraine Ransley, Carol Powell, Janet Blake, Jenna Plank, and Annette Crowley-Luke from the immunoassay laboratory. We thank our colleagues at the National Reference Laboratory for Bacterial Meningitis, Netherlands, for allowing us to present their recent Hib incidence figures.

Contributors: JM, MPES, and MER obtained and analysed disease incidence data. CLT obtained and analysed seroepidemiological data. All authors contributed to study design and preparation of the final manuscript. MER is guarantor.

Competing interests: MER and MPES have received research grants from vaccine manufacturers. JM was previously employed in an academic research post that was funded by a vaccine manufacturer. MER, MPES, and JM have all received funds from vaccine manufacturers to attend conferences and meetings.

Funding: The seroepidemiology component of this study was partially funded by the Public Health Laboratory Service Small Scientific Initiatives fund. Surveillance for invasive Hib infections is part of the core work of the Health Protection Agency's Communicable Disease Surveillance Centre and Haemophilus Reference Unit.

Ethical approval: The Health Protection Agency has approval under Section 60 of the Health and Social Care Act to process confidential information about patients for the purposes of monitoring the efficacy and safety of vaccination programmes.

1 Health Protection Agency. Quarterly communicable disease reports on the COVER programme for childhood immunisation. wwwhpa.org.uk/ infections/topics_az/vaccination/cover_cdr.htm (accessed 11 Aug 2004)

2 Trotter CL, Ramsay ME, Slack MPE. Rising incidence of Haemophilus influenzae type b disease in England and Wales indicates a need for a second catch-up vaccination campaign. Commun Dis Public Health 2003; 6:55-8.

3 Ramsay ME, McVernon J, Andrews N, Heath PT, Slack MPE. Estimating Hib vaccine efficacy in England and Wales using the screening method. $J$ Infect Dis 2003:188:481-5.

4 Trotter CL, McVernon J, Andrews NJ, Burrage M, Ramsay ME. Antibody to Haemophilus influenzae type $\mathrm{b}$ after routine and catch-up vaccination. Lancet 2003;361:1523-4.

5 McVernon J, Andrews NJ, Slack MPE, Ramsay ME. Risk of vaccine failure after Haemophilus influenzae type b (Hib) combination vaccines with acellular pertussis. Lancet 2003;361:1521-3.

6 Sarangi J, Cartwright K, Stuart J, Brookes S, Morris R, Slack M. Invasive Haemophilus influenzae disease in adults. Epidemiol Infect 2000;124: $441-7$
Osborne K, Gay N, Hesketh L, Morgan-Capner P, Miller E. Ten years of serological surveillance in England and Wales: methods, results, implications and action. Int J Epidemiol 2000·29:362-8.

8 Phipps DC, West J, Eby R, Koster M, Madore DV, Quataert SA. An ELISA employing a Haemophilus influenzae type b oligosaccharide-human serum albumin conjugate correlates with the radioantigen binding assay. J Immunol Methods 1990;135:121-8.

9 National Statistics Online. Birth statistics: births and patterns of family building England and Wales (FM1). www.statistics.gov.uk/StatBase Product.asp? $\mathrm{vlnk}=5768 \&$ Pos $=3 \&$ ColRank $=1 \&$ Rank $=144 \quad$ (accessed 7 Jul 2004).

10 McVernon J, Howard AJ, Slack MPE, Ramsay ME. Long term impact of vaccination on Haemophilus influenzae type b (Hib) carriage in the United Kingdom. Epidemiol Infect 2004;132:765-7.

11 Slack MPE, Azzopardi HJ, Hargreaves RM, Ramsay ME. Enhanced surveillance of invasive Haemophilus influenzae disease in England, 1990 to 1996: impact of conjugate vaccines. Pediatr Infect Dis 1998;17:S204-7

12 Kelly H, Riddell MA, Gidding HF, Nolan T, Gilbert GL. A random cluster survey and a convenience sample give comparable estimates of immunity to vaccine preventable diseases in children of school age in Victoria, Australia. Vaccine 2002;20:3130-6.

13 Boisvert PL. Familial epidemiology of Haemophilus influenzae, type b, infections. Am J Dis Child 1948;427-8.

14 Michaels RH, Norden CW. Pharyngeal colonization with Haemophilus influenzae type b: a longitudinal study of families with a child with menin

Barbour ML, Mayon-White RT, Coles C, Crook DWM, Moxon ER. The Barbour ML, Mayon-White RT, Coles C, Crook DWM, Moxon ER. The
impact of conjugate vaccine on carriage of Haemophilus influenzae type impact of conjugate vaccine

b. J Infect Dis 1995;171:93-8. EAM. Return of Haemophilus influenzae type b infections. Lancel 2003;361:1563.

17 European Union Invasive Bacterial Infections Surveillance Network Invasive Haemophilus influenzae in Europe, 2002. www.euibis.org documents/2002 hibreport.pdf (accessed 7 Jul 2004).

18 Glode MP, Halsey NA, Murray M, Ballard TL, Barenkamp S. Epiglottitis in adults: association with Haemophilus influenzae type b colonization in adults: association with Haemophilus influenzae type

19 Leino T, Auranen K, Makela PH, Kayhty H, Takala AK. Dynamics of natural immunity caused by subclinical infections, case study on Haemophilus influenzae type b (Hib). Epidemiol Infect 2000;125:583-91.

20 Added protection planned against Hib. Booster vaccine to be offered following increase in cases. Press release 2003/0071. London: Department of Health, 18 February 2003

21 Ramsay ME, Andrews NJ, Trotter CL, Kaczmarski EB, Miller E. Herd immunity from meningococcal serogroup $\mathrm{C}$ conjugate vaccination in England: database analysis. BMJ 2003;326:365-6.

22 Whitney CG, Farley MM, Hadler J, Harrison LH, Bennett NM, Lynfield R, et al. Decline in invasive pneumococcal disease after the introduction of protein-polysaccharide conjugate vaccine. New Engl J Med protein-polysaccharide

(Accepted 19 July 2004)

\section{Bullying among doctors in training: cross sectional questionnaire survey}

Elisabeth Paice, Maryanne Aitken, Anita Houghton, Jenny Firth-Cozens

London Deanery,

London

WC1N 1 DZ

Elisabeth Paice

dean director

Maryanne Aitken

project manager

Anita Houghton

associate dean

Jenny Firth-Cozen special adviser

Correspondence to: E Paice

epaice@

londondeanery.ac.uk

BMJ 2004;329:658-9
Workplace bullying is associated with stress, depression, and intention to leave. It is an important issue for the health service because of its potential impact on staff health, retention, and patient care. ${ }^{12}$ In a recent survey of UK doctors in training, 37\% said they had been bullied during the past year. ${ }^{3}$ To understand the problem better, we investigated how commonly doctors in training experienced persistent and serious bullying, who were the sources of this behaviour, and what action was taken to deal with it.

\section{Participants, methods, and results}

We conducted a cross sectional questionnaire survey of doctors in training in London north of the Thames, using electronic survey units followed up by postal questionnaire, as described previously. ${ }^{4}$ Our sample was defined as all trainees available at the time of the survey in participating trusts. The survey included four questions on bullying. The stem question, derived from one used by Hicks, ${ }^{2}$ was: "In this post, have you been subjected to persistent behaviour by others which has eroded your professional confidence or self esteem?" The analysis of differences between group frequencies was calculated using the $\chi^{2}$ test with adjusted residuals.

All 21 hospital trusts and six of the seven community and mental health trusts took part. The response rate overall was $72 \%(2730 / 3779)$, with rates for individual trusts ranging from $40 \%$ to $98 \%$. The stem question was answered by $2673 / 2730 \quad(98 \%)$ of respondents, three of whom did not record their sex and five of whom did not record their grade. These

Five tables of further data about the respondents and their responses are on bmj.com

This article was posted on bmj.com on 15 July 2004: http://bmj.com/cgi/ doi/10.1136/bmj.38133.502569.AE 